\title{
DESIGNING OF CATAMARAN HULL SPINE BEAM
}

\author{
Alexander Janushevskis, Jaymin-Vrajlal Sanchaniya, Sanjay-Rajni Vejanand \\ Riga Technical University, Latvia \\ jaymin.sanchaniya@rtu.lv, sanjay.vejanand@rtu.lv
}

\begin{abstract}
Demand for improvement in shipbuilding is increasing and due to this many manufacturing industries are developed rapidly and come up with very advanced designs and development in this field. Specifically, there is a good scope for improvements in the structure of the Class 6 catamaran hull spine beam. Usually, the multihull catamaran operates beyond its design specifications and due to unforeseen winds, waves, and deteriorating weather, the hull spine beam faces more impacts what leads to failure and weakening after decades of sustained operation. The analysis results obtained in this paper show great improvement in the design of the catamaran hull spine beam, which would give an idea of overall improvement in catamaran design and its performance. The design methods for developing catamaran and bulkheads are considered in the study. The whole analysis is performed on ANSYS software by following step by step simulation procedure using the FEM methodology. At first, simulation of simple the 3D model without considering bulkheads is done and results are observed. In the next steps, bulkheads are added to the spine beam at equal distance in a sequence of 1,3,5,7, and 9 along the beam. The obtained results confirm that adding more and more bulkheads makes the structure bulky, but adding it up to a certain level helps reduce maximum Von-Mises stress by $75.9 \%$ and total deformation of the spine beam by $70.9 \%$, which makes the structure more rigid and essentially improves the strength of the catamaran hull spine beam.
\end{abstract}

Keywords: catamaran, spine beam, finite element analysis, strength optimization.

\section{Introduction}

The catamaran is a type of ship with two parallel hulls equal in size which are connected to the beam. The wide beam of the catamaran helps provide geometric stability to this watercraft.

Usually, the catamaran operates outside its design parameters or capacity, due to its characteristics. Moreover, the external parameters such as wind, unpredictable waves and deteriorating weather make high damage to the catamaran hull. In such situations, the catamaran hull spine beam experiences more impact from all sides, whereas the spine beam is made up of composite materials and is designed considering static load to be applied from particular direction only. This results in the basic failure mechanism of the hull spine beam. The most common are fatigue failure, cracking, delamination between piles, fibre breakage of solid laminates and de-bonding of the resin-fibre interface [1-3]. It is very important to investigate and improve the design of catamaran hull spine beam to avoid such type of failures.

The maritime sector, like many other sectors, is seeing an increase in the usage of composite materials and structures. Existence of fabrication defects in a catamaran is likely to result in structural failure during operation loads. The system must be designed in such a manner that any harm sustained during the structure's regular operating life would not result in its failure until the damage is found. In comparison to this conventional method, the primary additional difficulty associated with a harm tolerance-based architecture approach is the need for more accurate knowledge regarding the structure's behaviour. In other terms, if we expect damage to accumulate over the structure's service life, we must ensure that we can precisely predict how the damage will occur over time and then schedule an adequate plan. A single load can produce several interacting failure modes in composite systems, and in certain situations, initial defects and damage in composite materials expand faster than in metals. It is well known that composite materials/structures may be subjected to stress analysis. On the other hand, failure analysis of composite materials is a complex and difficult task. Finite element analysis is often used to assess the failure evolution of composite materials/structures [4-6].

Several recent investigations have been made to improve the efficiency of massive watercraft structures using construction improvements, work [7] has shown that the Deck Pockets (bulkheads) are to be mounted in the vertical lines wherever necessary, and the structures at the head and tail are employed to evenly spread the load.

Expanded results from study [8] show that the quest for composite device crash resistant designs encompasses the idea of subfloor designs (such as bullheads) that have the same goals as other subfloor energy absorbing and weight saving properties as composite structures in air. A follow-up research [9] on the methodology of minimising the vertical corrugated bulkhead weight of a chemical tanker was 
completed, which showed an estimated weight reduction of 9.3\%. Using laboratory experiments and theoretical analysis, researchers [10] demonstrate that overall active pressure generally does not surpass the calculated active pressure exerted on bulkheads, but instead focuses on and divides between the anchors and in the region of the pile.

In studies $[11 ; 12]$ hull forms and hydrodynamic characteristics are investigated to assess whether numerical approaches may be applied to the initial design of a catamaran. Results show that these methods benefit the initial design, especially for the initial design of catamaran hull spine beam.

The main goal of this work is to design a spine hull beam by adding bulkheads, as the spine beam provides structural support to the whole catamaran. The catamaran is consisting of a $\mathrm{V}$ shaped beam that connects two hulls and adding bulkheads to this beam can increase the strength, load bearing capacity of the catamaran.

\section{Structure design of catamaran hull spine beam}

Preparing a proper model is very important in any analysis, as any uneven or open surface within the geometric model may result in failure or poor meshing that directly will impact the study results. Hence, it is very important to design a clean and smooth model for good quality analysis. Also, model should be made as simple as possible by removing complex unwanted geometries if any in the model to reduce computational time and achieve good quality results.

The catamaran hull (see Fig. 1) is of $8.5 \mathrm{~m}$ width, $2.7 \mathrm{~m}$ height and $19 \mathrm{~m}$ long. The catamaran hull possesses $0.03 \mathrm{~m}$ of all over thickness.

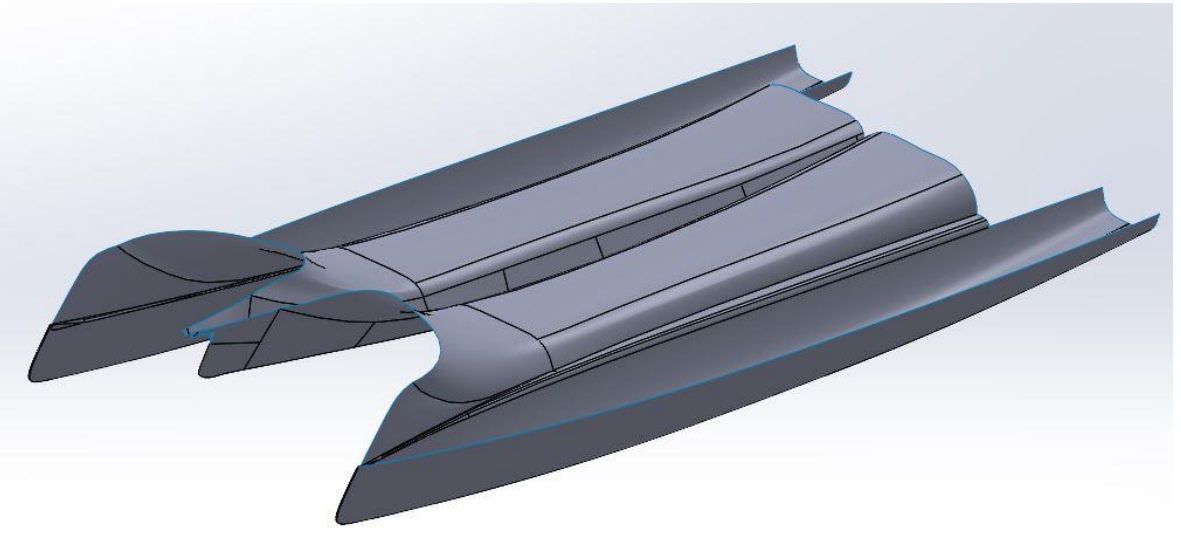

Fig. 1. Catamaran hull design (3D model)

\section{Analysis of catamaran hull spine beam}

The model designed in SolidWorks is imported and further simulation procedures are followed in the ANSYS software [13]. The detail simulation steps are described below.

In practical design, the structure of catamaran is made up of composite material [14;15]. Hence, composite material is also assigned to the study model for the analysis. The material properties of assigned composite material are shown in Table 1.

Effective properties of composite material

\begin{tabular}{|c|c|}
\hline Property & Value \\
\hline Young's Modulus & $20000 \mathrm{MPa}$ \\
\hline Poisson's Ratio & 0.3 \\
\hline Bulk Modulus & $16667 \mathrm{MPa}$ \\
\hline Shear Modulus & $7692.3 \mathrm{MPa}$ \\
\hline Compressive Yield Strength & $118 \mathrm{MPa}$ \\
\hline Tensile Yield Strength & $930 \mathrm{MPa}$ \\
\hline
\end{tabular}

Table 1

Meshing is a very important step in any analysis as the quality of mesh will directly affect the results of the analysis. Higher the quality of mesh, higher the possibility to achieve more accurate results. 


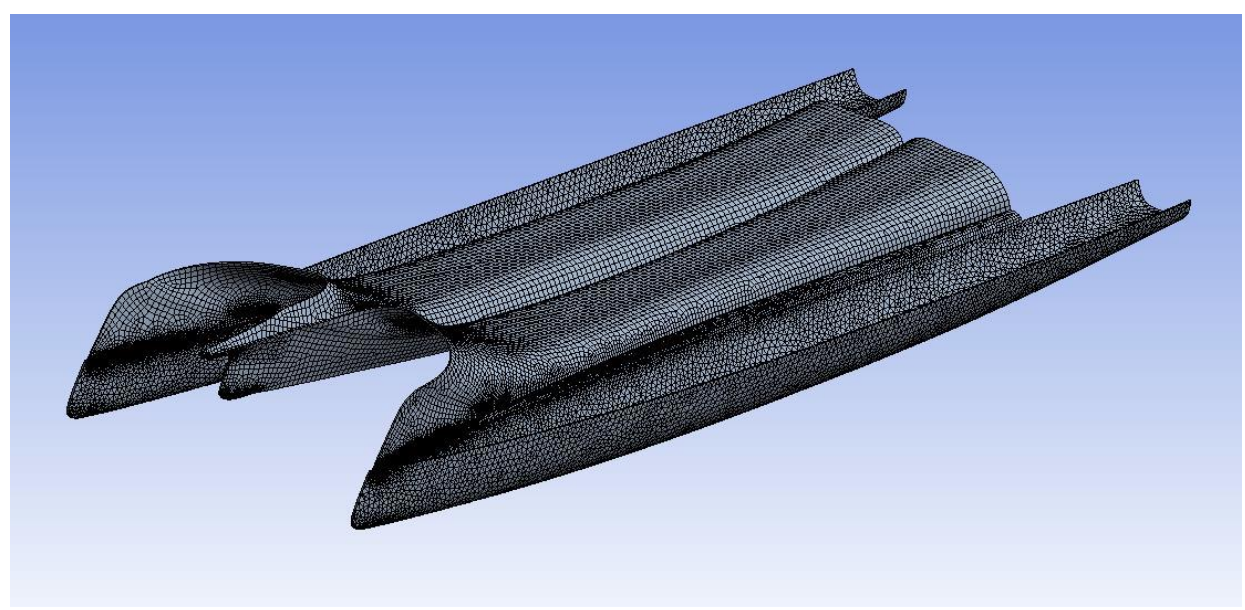

Fig. 2. Meshing of catamaran hull

Figure 2 shows the catamaran model after generating mesh, proper-uniform mesh generation is visualized over the whole model. Quadrilaterals type of mesh is used to generate mesh.

The present catamaran hull design is developed to withstand load up to 20 tons from the top. Considering general situation either stationary or floating in water, the catamaran hull will be submerged into the water till the water line. Hence, the bottom portion of the hulls are fixed, and the load is applied from the top, as shown in Figure 3.

The results are evaluated in form of Von Mises stress and total deformation.

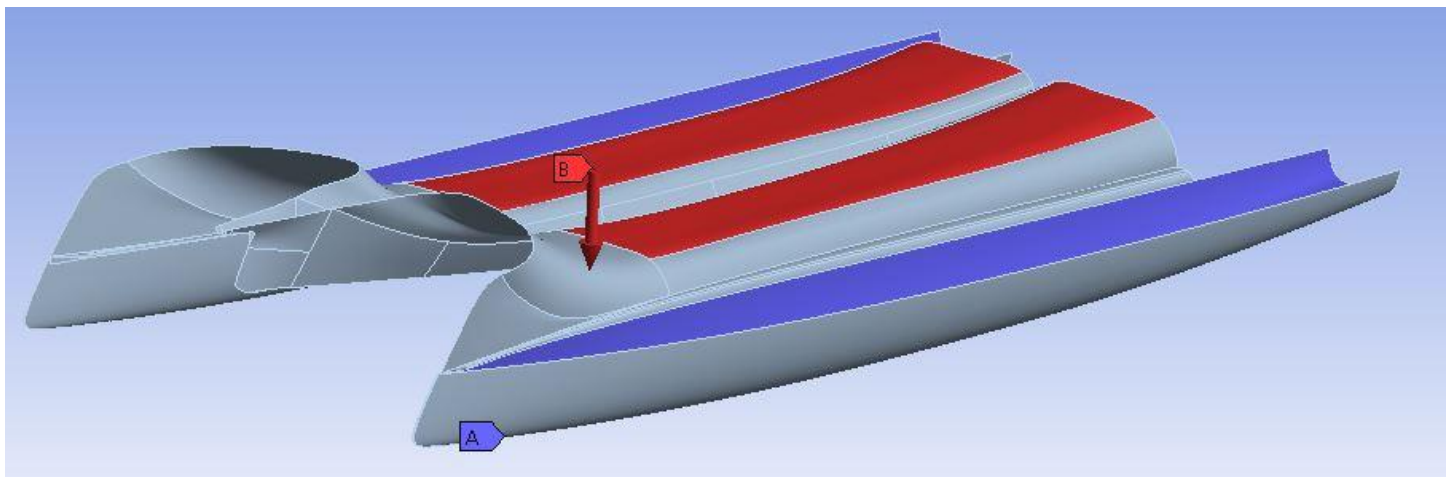

Fig. 3. Fixed support location (A) and location of applied force (B)

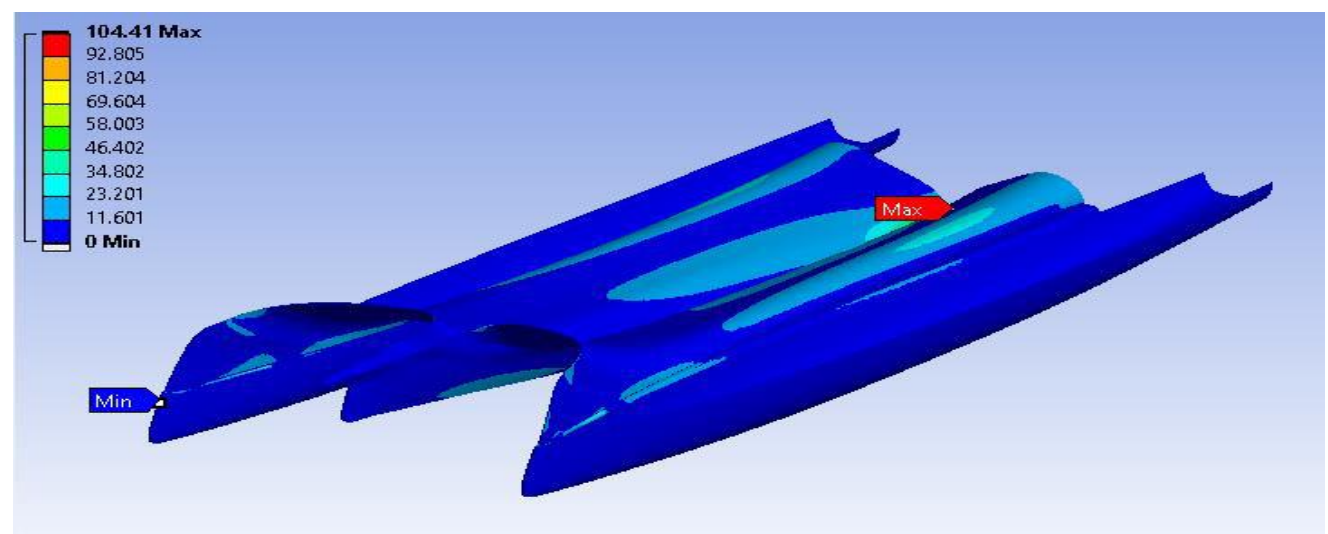

Fig. 4. Von-Mises stresses

As it can be observed from Figure 4, maximal Von-Mises stress is acting at the middle portion of the structure because of the hollow $\mathrm{V}$ section in the middle. The obtained value of maximum stress is 104.41 MPa, acting at the central back end of the spine beam. As observed in Figure 5, mainly the central hollow portion of the beam is experiencing the maximum deformation of $154.51 \mathrm{~mm}$. 


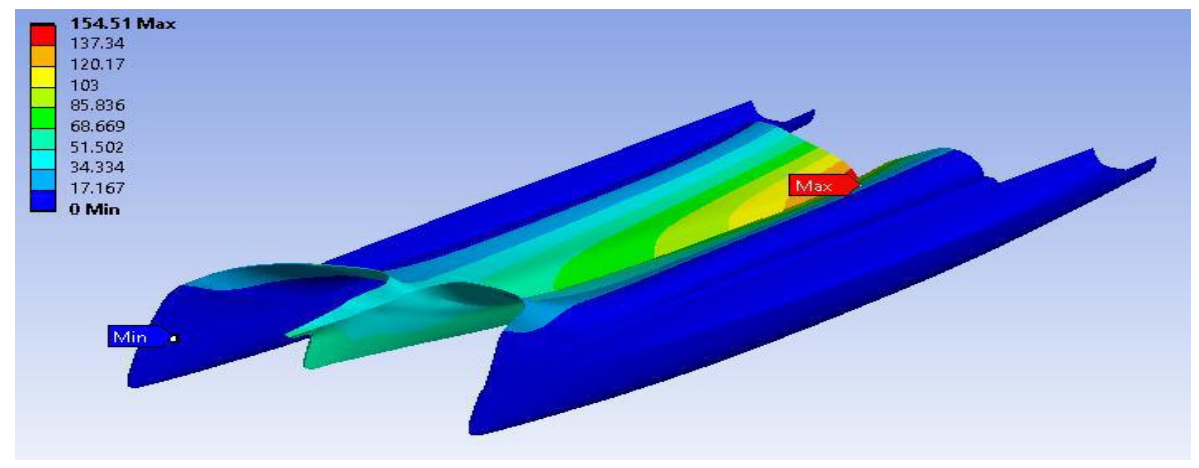

Fig. 5. Total deformations

\section{Analysis of spine beam with bulkheads}

As we have observed from the analysis results in section 4, the weaker portion in the catamaran hull structure is the central spine beam due to its hollow section, as the maximum stress is found at the spine beam. Moreover, deformation is also occurring mainly towards the spine beam. Adding bulkheads could improve the results and strength of the structure and further analysis with bulkheads will help understand it more closely. Here bulkheads are added in the sequence of 1,3,5,7 and 9 respectively and at the end a special case is added where the simple structure is developed on the catamaran hull to give a more practical approach.

Bulkheads (Fig. 6) are added in a hollow V shaped beam as shown below in Figure 7. The same material properties are given to the bulkheads as assigned to the hull structure. Bulkheads are designed in such a way that they fit properly to the central hollow section and joined rigidly with catamaran hull.

Figure 7 below shows the beam structure with added bulkheads. The analysis is made individually starting with single bulkhead until 9 bulkheads and the results are compared discussed at the end. The same procedures are followed for the analysis as performed in previous simulation done without bulkheads, the only difference here will be that the applied force is divided into the top surface of bulkheads with the hull structure.

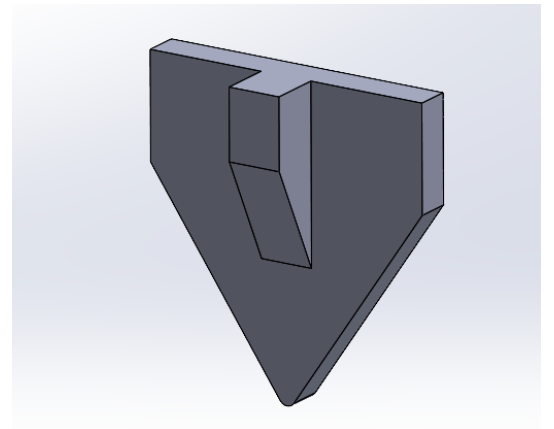

Fig. 6. Bulkhead

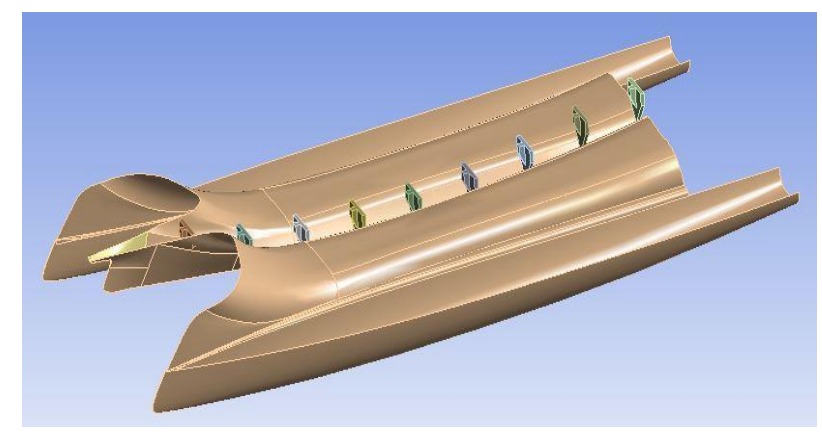

Fig. 7. Catamaran hull spine beam with bulkheads

While increasing in number, the bulkheads are added to the spine beam at equal distances from the centre.

\section{Results}

The numerical results after adding each bulkhead are shown in Table 2. From the obtained results it is quite clear that adding 7 bulkheads provides the best results. The maximum Von-Mises stress and total deformation in case of 7 bulkheads are shown in below Figure 8 and 9, respectively.

Figure 10 and Figure 11 show that through the spine beam and around the beam maximum VonMises stress found is $17.4 \mathrm{MPa}$ and observing close towards the centre of spine beam it is increasing to 18.2 MPa. Therefore, in Figure 8 the cross section of spine beam is considered, which is showing maximum Von-Mises stress $25.16 \mathrm{MPa}$ and the same for deformation as well. The individual results after adding each bulkhead are mentioned in Table 2 . 


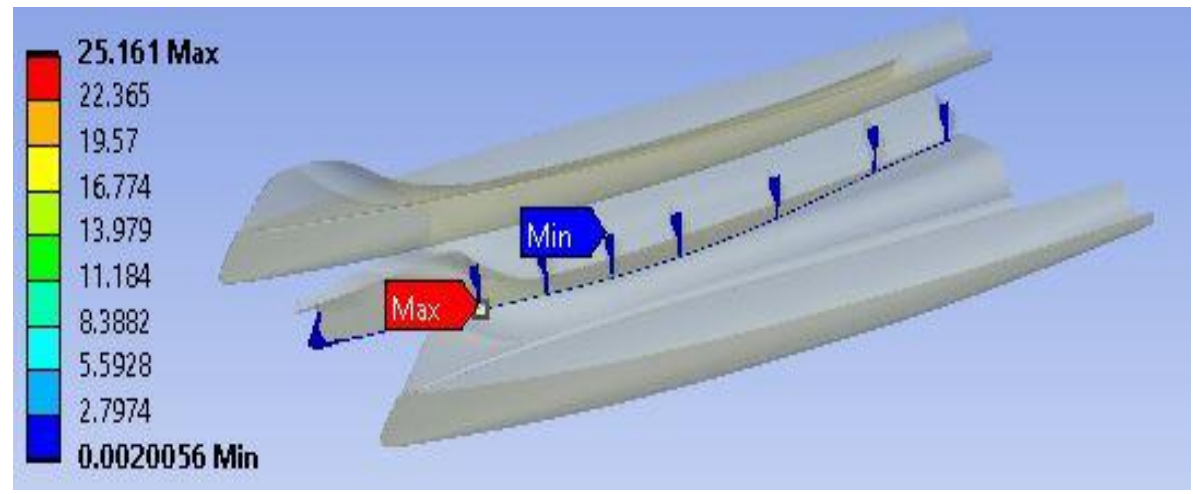

Fig. 8. Maximum Von-Mises stress in spine beam with 7 bulkheads

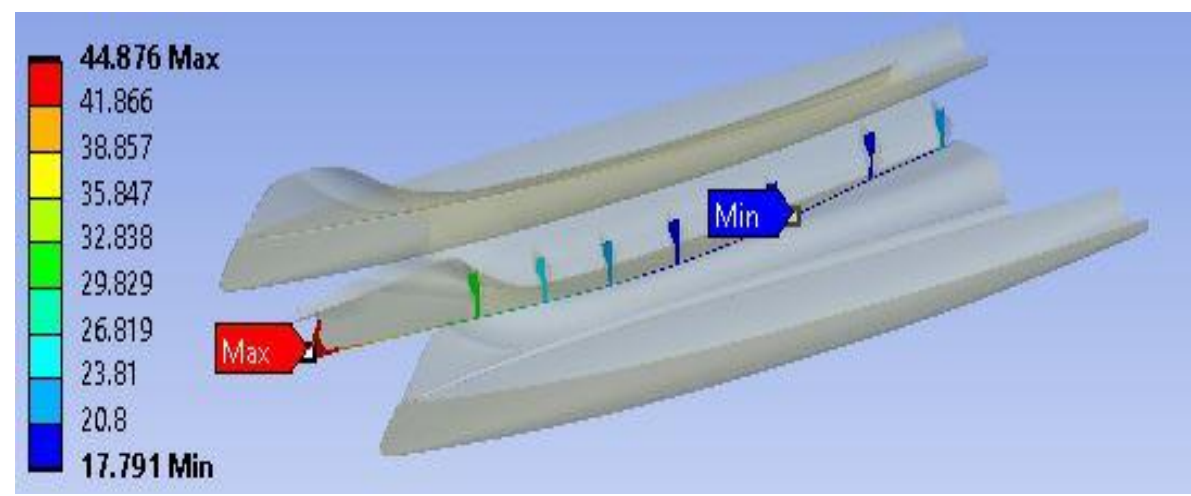

Fig. 9. Total deformation in spine beam with 7 bulkhead

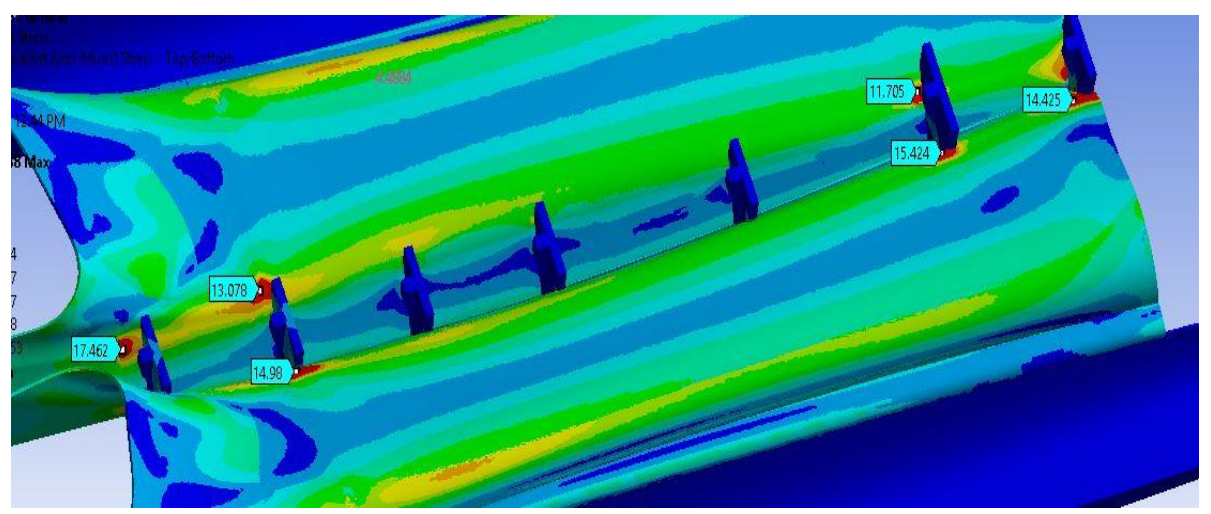

Fig. 10. Von-Mises stress through spine beam

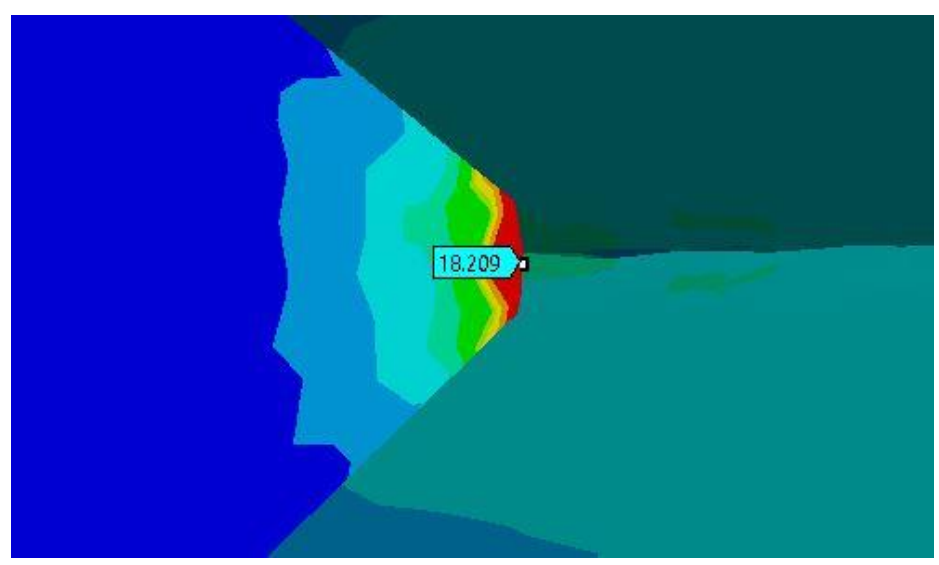

Fig. 11. Von-Mises stress in centre of spine beam 


\section{Comparison of results}

\begin{tabular}{|c|c|c|}
\hline Number of bulkheads & Max. Von-Mises stress, MPa & Max. total deformation, mm \\
\hline 0 & 104.41 & 154.51 \\
\hline 1 & 98.759 & 147.78 \\
\hline 3 & 64.17 & 69.59 \\
\hline 5 & 51.75 & 61.33 \\
\hline 7 & 25.16 & 44.88 \\
\hline 9 & 52.55 & 61.62 \\
\hline
\end{tabular}

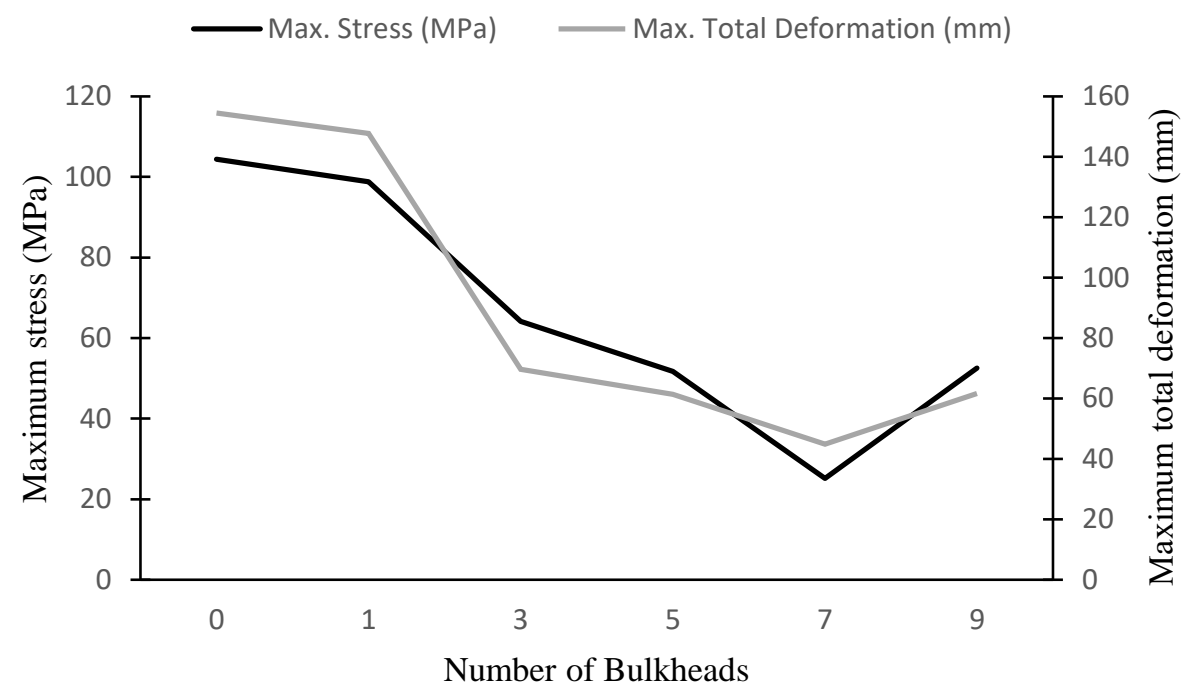

Fig. 12. Maximum stress and maximum total deformation vs the number of bulkheads

Comparing the results mentioned in Table 2 and Figure 12, it can be concluded that adding 7 bulkheads provides the best results with the lowest stress and deformation. Also, we can observe that maximum stress and total deformation decreased gradually with added number of bulkhead from 1 to 7 , but after adding 9 bulkheads, the stress and deformation again increases, which indicates that it is really important to add a specific number of bulkheads to obtain optimum results, as adding more bulkheads after a certain number also shows poor results.

\section{Conclusions and Discussion}

1. Predicting stress and deformation in the catamaran spine hull beam is a complex challenge that should not be pursued without the use of software-based simulation. FEM-based tech, such as ANSYS, is extremely useful for achieving results in a limited amount of time. ANSYS simplifies simulation for complex geometry, solves complex mathematical models quickly, and produces accurate results.

2. Fig. 12 depicts a graph of the effect of inserting bulkheads to the spine beam, with the vertical axis representing the value and the horizontal axis representing the number of bulkheads. There is not much of a change in maximal tension in the spine beam after the first bulkhead, but when we added the fifth bulkhead, it fell to about $64 \mathrm{MPa}$ and continued for the next seven bulkheads. The first bulkheads had no effect on overall deformation, but it changed drastically after the third bulkhead was added, and it continues to decline dramatically until at least seven bulkheads are added. The maximum stress was reduced by $75.9 \%$ and deformation was reduced by $70.9 \%$ in this case.

3. Adding bulkheads up to a certain limit yields good outcomes, but adding more bulkheads will make the system heavy and have detrimental effects on the whole structure. Simulations are more costeffective and efficient, if performed early in the design process to provide a stronger system.

4. The obtained results must be considered as the first step, where significance of bulkhead positioning is shown. It is necessary to take into account material peculiarities in detail, as well as different load cases due to dynamic excitation acting on catamaran. It is planned to use specific numerical 
optimization technics and metamodeling methods to seek the optimal position and number of bulkheads.

\section{Acknowledgments}

This research is funded by the Latvian Council of Science, project "Creation of design of experiments and metamodeling methods for optimization of dynamics of multibody 3D systems interacting with bulk solids and fluids", project No. lzp-2018/2-0281.

\section{References}

[1] McKenzie P., Meares J., Cull H. Dream Weaver flooding due to structural failure of the hull, Hauraki Gulf The Transport Accident Investigation Commission, Wellington, New Zealand, 2015.

[2] Lamb T. Ship Design and Construction. New Jersey: Society of Naval Architects and Marine Engineers, 2003-2004.

[3] Dasari N., Srinivas V. G. Design of Catamaran Ship Main Deck and Bulkhead to Withstand the Crane Load. International Journal of Engineering Research and Development, vol. 8, No. 3, 2013, pp. 31-41.

[4] Oterkus E., Diyaroglu C., De Meo D., Allegri G. Fracture modes, damage tolerance and failure mitigation in marine composites. In: Marine Applications of Advanced Fibre- Reinforced Composites. Woodhead Publishing Series in Composites Science and Engineering: No 63. Edited by J. Graham-Jones and J. Summerscales. Elsevier. 2016, pp. 79-102.

[5] Shivakumar, K., Chen, H. Structural performance of eco-core sandwich panels. In: Daniel, I.M., Gdoutos, E.E., Rajapakse, Y.D.S. (Eds.) Major Accomplishments in Composite Materials and Sandwich Structures. Springer, The Netherlands. 2009, pp. 381-406.

[6] Zenkert, D. Damage tolerance of naval sandwich panels. In: Daniel, I.M., Gdoutos, E.E., Rajapakse, Y.D.S. (Eds.), Major Accomplishments. Springer, The Netherlands. 2009, pp. 279-303.

[7] Fanchun L., Zang C., Laxmin L. Dejun J. Design and simulation analysis of trimaran bulkhead based on topological optimization. Elsevier, vol. 191, 2019, pp. 1-20.

[8] Yong Bai, Wei-Liang Jin. Marine Structural Design. Second Edition. Elsevier, ISBN: 978-0-08099997-5, 2016.

[9] Ko D. E., Shin S. A study on minimum weight design of vertical corrugated bulkheads for chemical tankers. International Journal of Naval Architecture and Ocean Engineering, vol. 10, No. 2, 2018, pp. 180-187.

[10] Tsinker G. P. Anchored Sheet Pile Bulkheads: Design Practice. Journal of Geotechnical Engineering, vol. 110, No. 8, 1984, pp. 1170-1178.

[11] Lee S. H., Lee Y. J., Lee J.W. Hull Form Design of a Passenger Catamaran For Operation in the Yellow Sea Region. Practical Design of Ships and Other Floating Structures, vol. 1, No. 1, 2001, pp. 83-89.

[12] Okada, Tetsuo, Suzuki, Katsuyuki, et. al. Practical Design of Ships and Other Floating Structures, vol. 2, Tokohama, Japan: Springer, 2019.

[13] ANSYS, “ANSYS User Manual,” ANSYS, [online] [10.05.2021] Available at: https://www.ansys.com/

[14] Lyashenko I. et. al. Manufacturing of Amber Particles Suitable for Composite Fiber Melt Spinning. The Journal of Latvian Academy of Sciences, vol. 70, 2016, pp. 51-57.

[15] Lyashenko I. et. al. Application of amber filler for production of novel polyamide composite fiber. Textile Research Journal, vol. 86 (20), 2016, pp. 2127-2139. 